\title{
Recent Developments on Representation of Numerical Data by a Polynomial Curve
}

\author{
Dhritikesh Chakrabarty \\ Department of Statistics, Handique Girls' College, Guwahati, Assam, India, \\ dhritikesh.c@ rediffmail.com , dhritikeshchakrabarty@gmail.com
}

\begin{abstract}
.
Recently, some studies have been made on accurate mathematical representation of numerical data by polynomial curve. Some formulae have been derived, for representation of numerical data by polynomial curve, from some of the existing formulae for numerical interpolation. Moreover, some methods have been composed of for the same purpose. This paper describes an overview of the developments of these methods/formulae for representing numerical data on a pair of variables by a polynomial curve.
\end{abstract}

Key words: 1; Pair of variables 2;polynomial curve 3; representation of numerical data 4;method \& formula.

\section{Introduction:}

The problems of interpolation, extrapolation, forecasting/projection etc. are solved, in many situations, by fitting a mathematical curve to the available numerical data on variable(s) under study. The fitting of a mathematical curve to numerical data is nothing but an approximate representation of the data by the curve in the sense of minimization of error. Recently, some studies have been made on accurate mathematical representation of numerical data by polynomial curve. Some formulae have been derived, for representation of numerical data by

polynomial curve, from some of the existing formulae for numerical interpolation. Moreover, some methods have been composed of for the same purpose.

Interpolation, which is the process of computing intermediate values of a function from the set of given values of the function [Erdos \& Turan (1938) ; Hummel (1947) et al], plays significant 
role in numerical research almost in all branches of science, humanities, commerce and in technical branches. A number of interpolation formulas namely Newton's Forward Interpolation formula, Newton's Backward Interpolation formula, Lagrange's Interpolation formula, Newton's Divided Difference Interpolation formula, Newton's Central Difference Interpolation formula, Stirlings formula, Bessel's formula and some others are available in the literature of numerical analysis [Neale \& Sommerville (1924) ; Jan (1930) ; Hummel (1947) ; Jordan (1965) ; Abramowitz (1972) ; Bathe \& Wilson (1976) ; Conte \& Boor (1980) ; Kendall (1989) ; Vertesi (1990) ; Kincard \& Ward (1991) ; Gerald \& Wheatley (1994) ; Scarborough (1996) ; Robert \& Sandra (2000) ; Chapra \& Canale (2002) ; Endre \& David (2003) ; John (2004) et al)].

In case of the interpolation by the existing formulae, the value of the dependent variable corresponding to each value of the independent variable is to be computed afresh from the used formula putting the value of the independent variable in it. That is if it is wanted to interpolate the values of the dependent variable corresponding to a number of values of the independent variable by a suitable existing interpolation formula, it is required to apply the formula for each value separately and thus the numerical computation of the value of the dependent variable based on the given data are to be performed in each of the cases. In order to get rid of these repeated numerical computations from the given data, one can think of an approach which consists of the representation of the given numerical data by a suitable polynomial and then to compute the value of the dependent variable from the polynomial corresponding to any given value of the independent variable. However, there is necessary of method/formula for representing a given set of numerical data on a pair of variables by a suitable polynomial. Recently, some studies have been made on accurate mathematical representation of numerical data by polynomial curve [Das \& Chakrabarty $(2016 a, 2016 b, 2016 c, 2016 d, 2016 e, 2016 f)$, Chakrabarty $(2016 a, 2016 b$, 2016c)]. Some formulae have been derived, for representation of numerical data by polynomial curve, from some of the existing formulae for numerical interpolation. Moreover, some methods have been composed of for the same purpose independently. For this purpose Chakrabarty has searched for one method/formula for representing a given set of numerical data on a pair of variables by a suitable polynomial. Chakrabarty has derived another formula for the same purpose. Das \& Chakrabarty have derived four formulae for the same purpose [Das \& Chakrabarty $(2016 a, 2016 b, 2016 c, 2016 d, 2016 e, 2016 f)]$. They have derived the first formula [Das \& Chakrabarty (2016a)] from formula from Lagrange's Interpolation formula 
[Echols (1893) ; Corliss (1938) ; Hummel (1947) ; Traub (1964) ; Jardan (1965) ; Quadling (1966) ; Mills (1977) ; Kendall (1989) ; Kincard \& Ward (1991) ; Revers \& Michael (2000) ; Endre \& David (2003) ; Jan (2004) ; Nasrin (2010) et al]. The second formula [Das \& Chakrabarty (2016b)] has been derived from Newton's Divided Difference Interpolation Formula [Herbert (1962); Whittaker \& Robinson (1967a , 1967b) ; De Boor (2003) et al]. The third one (Das \& Chakrabarty , 2016c) has been derived from Newton's Forward Interpolation formula [Erdos \& Turan (1938); Whittaker \& Robinson (1967a); Nasrin (2010) et al] and the fourth one (Das \& Chakrabarty $(2016 d$ ) has been derived from Newton's Backwards Interpolation formula [Erdos \& Turan (1938) ; Hummel (1947) ; Jardan (1965) ; Whittaker \& Robinson (1967a) ; Kendall (1989) ; Kincard \& Ward (1991) ; Endre \& David (2003) ; Jan (2004) et al]. Later on one method has been constructed by Chakrabarty $(2016 a, 2016 b, 2016 c)$ for representing a given set of numerical data on a pair of variables by a suitable polynomial. The method based on two numerical operations namely finite difference operation and ratio operation [Gertrude(1954) ; Herbert (1962) ; Jordan (1965) ; Dokken \& Lyche (1979) ; Fred (1979) ; Jeffreys \& Jeffreys (1988) ; Lee (1989) ; Chwaiger (1994) ; De Boor (2003) ; Endre \& David (2003) ; Floater (2003) et al]. In another study, two methods have been composed by Das \& Chakrabarty $(2016 e, 2016 f)$ for the same purpose. The two methods are based on the inversion of a square matrix. The first one is based on matrix inversion from Cayley-Hamilton theorem [Cayley $(1858,1889)$; Hamilton $(1853,1862,1864 a, 1864 b)$ et al] while the second one is on matrix inversion by elementary transformation of matrix [Cayley (1858, 1889 et al); Hamilton (1889) et al]. This paper describes an overview of the developments of these methods/formulae for representing numerical data on a pair of variables by a polynomial curve with numerical examples in order to show the application of the methods/formulae developed.

\section{Method/Formula for Representation of Numerical Data by Polynomial Curve:}

Let us consider a function

$$
y=f(x)
$$

and let

$$
y_{0}=f\left(x_{0}\right), y_{1}=f\left(x_{1}\right), y_{2}=f\left(x_{2}\right), \ldots \ldots \ldots \ldots \ldots \ldots \ldots, y_{n}=f\left(x_{n}\right)
$$

be the values of $y=f(x)$ corresponding to the values 


$$
x_{0}, x_{1},\left(x_{2}, \ldots \ldots \ldots \ldots \ldots \ldots \ldots \ldots, x_{n}\right.
$$

of $x$.

Then,

$$
\left(x_{0}, y_{0}\right),\left(x_{1}, y_{1}\right),\left(x_{2}, y_{2}\right) \ldots \ldots \ldots \ldots \ldots \ldots \ldots \ldots \ldots\left(x_{n}, y_{n}\right)
$$

be the set of $(n+1)$ pairs of values of $x$ and $y$.

\section{1. Formula Obtained from Newton's Forward Interpolation Formula:}

Let the values of the independent variable $x$ be at equal interval $h$ i.e.

$$
x_{i+1}-x_{i}=h \text { for all } i
$$

Further let

$$
u=\frac{x-x_{0}}{h}
$$

Then Newton's forward interpolation formula is

$$
\begin{aligned}
& f(x)=f\left(x_{0}\right)+u \Delta f\left(x_{0}\right)+\frac{u(u-1)}{2 !} \Delta^{2} f\left(x_{0}\right)+\frac{u(u-1)(u-2)}{3 !} \Delta^{3} f\left(x_{0}\right)+ \\
& +\frac{u(u-1)(u-2) \ldots \ldots \ldots \ldots(u-n+1)}{n !} \Delta^{n} f\left(x_{0}\right),
\end{aligned}
$$

[Erdos \& Turan (1938) ; Whittaker \& Robinson (1967a) ; Nasrin (2010) et al].

This formula can be expressed as

$$
\begin{aligned}
f(x)=C_{0} & +C_{1}\left(x-x_{0}\right)+C_{2}\left(x-x_{0}\right)\left(x-x_{1}\right)+C_{3}\left(x-x_{0}\right)\left(x-x_{1}\right)\left(x-x_{2}\right)+ \\
& +C_{n}\left(x-x_{0}\right)\left(x-x_{1}\right)\left(x-x_{2}\right)\left(x-x_{3}\right) \ldots \ldots \ldots \ldots \ldots\left(x-x_{n-1}\right)
\end{aligned}
$$

where $\quad C_{i}=\frac{\Delta^{i} f\left(x_{0}\right)}{i ! h^{i}}, i=0,1,2,3$, $,(n-1), n$

Now, equation (2.1.2), can be expressed as

$$
f(x)=A_{0}+A_{1} x+A_{2} x^{2}+A_{3} x^{3}+\ldots \ldots \ldots \ldots \ldots \ldots \ldots \ldots+A_{n} x^{n}
$$

where

$$
\begin{aligned}
& A_{0}=C_{0}-C_{1} x_{0}+C_{2} x_{0} x_{1}-C_{3} x_{0} x_{1} x_{2}+C_{4} x_{0} x_{1} x_{2} x_{3}- \\
& +C_{n}(-1)^{n}\left(x_{0} x_{1} x_{2} x_{3} \ldots \ldots \ldots \ldots x_{n-1}\right), \\
& A_{1}=C_{1}-C_{2}\left(\sum_{\mathrm{i}=0}^{1} \mathrm{x}_{\mathrm{i}}\right)+C_{3}\left(\sum_{\mathrm{i}=0}^{1} \sum_{\mathrm{j}=1}^{2} \mathrm{x}_{\mathrm{i}} \mathrm{x}_{\mathrm{j}}\right)-C_{4}\left(\sum_{\mathrm{i}=0}^{1} \sum_{\mathrm{j}=1}^{2} \sum_{\mathrm{k}=2}^{3} \mathrm{x}_{\mathrm{i}} \mathrm{x}_{\mathrm{j}} \mathrm{x}_{\mathrm{k}}\right)+
\end{aligned}
$$




$$
\begin{aligned}
& (-1)^{n} C_{n}\left(x_{0} x_{1} x_{2} x_{3}\right. \\
& x_{n-2}+x_{0} x_{1} x_{2} x_{3} \\
& \left.x_{n-1}\right) \text {, } \\
& A_{2}=C_{2}-C_{3}\left(\sum_{\mathrm{i}=0}^{2} \mathrm{x}_{\mathrm{i}}\right)+C_{4}\left(\sum_{\mathrm{i}=0}^{2} \sum_{\mathrm{j}=1}^{3} \mathrm{x}_{\mathrm{i}} \mathrm{x}_{\mathrm{j}}\right)- \\
& (-1)^{n} C_{n}\left(x_{0} x_{1} x_{2} x_{3} \ldots \ldots \ldots \ldots x_{n-3}+x_{0} x_{1} x_{2} x_{3} \ldots \ldots \ldots \ldots x_{n-2}+\right. \\
& \left.x_{0} x_{1} x_{2} x_{3} \ldots \ldots \ldots \ldots x_{n-1}\right) \text {, } \\
& A_{3}=C_{3}-C_{4}\left(\sum_{\mathrm{i}=0}^{3} \mathrm{x}_{\mathrm{i}}\right)+\ldots \ldots \ldots . .+(-1)^{n} C_{n}\left(x_{0} x_{1} x_{2} x_{3} \ldots \ldots \ldots \ldots x_{n-4}+\right. \\
& \left.x_{0} x_{1} x_{2} x_{3} \ldots \ldots \ldots \ldots x_{n-3}+x_{0} x_{1} x_{2} x_{3} \ldots \ldots \ldots \ldots x_{n-2}+x_{0} x_{1} x_{2} x_{3} \ldots \ldots \ldots \ldots x_{n-1}\right) \text {, } \\
& A_{i}=C_{i}-C_{i+1}\left(\sum_{\mathrm{j}=0}^{\mathrm{i}} \mathrm{x}_{\mathrm{j}}\right)+\ldots \ldots \ldots \ldots \ldots+(-1)^{n-i} C_{n}\left(x_{0} x_{1} x_{2} x_{3} \ldots \ldots \ldots \ldots x_{n-i+1}\right. \\
& +x_{0} x_{1} x_{2} x_{3} \ldots \ldots \ldots \ldots x_{n-1} \\
& A_{n}=C_{n}
\end{aligned}
$$

Equation (2.1.3), with the coefficients

$$
A_{0}, A_{1}, A_{2}, A_{3}, \ldots \ldots \ldots \ldots \ldots \ldots \ldots, A_{n},
$$

as defined above, is one formula for representing a given set of numerical data on a pair of variables by a suitable polynomial .

Note: The formula is applicable for representing a given set of numerical data on a pair of variables by a suitable polynomial when the given values of the independent variable are at equal interval.

\section{An Example of Application of the Formula}

Example (2.1.1): The following table shows the data on total population of India corresponding to the years:

\begin{tabular}{|c|c|c|c|c|c|}
\hline Year & 1971 & 1981 & 1991 & 2001 & 2011 \\
\hline Total Population & 548159652 & 683329097 & 846302688 & 1027015247 & 1210193422 \\
\hline
\end{tabular}

Taking 1971 as origin and changing scale by 1/10, one can obtain the following table for independent variable $x$ (representing time) and $f(x)$ (representing total population of India): 
International Journal of Electronics and Applied Research (IJEAR), Vol.3 Issue 2, December, 2016

\begin{tabular}{|c|c|c|c|c|c|}
\hline Year & 1971 & 1981 & 1991 & 2001 & 2011 \\
\hline$x_{\mathrm{i}}$ & 0 & 1 & 2 & 3 & 4 \\
\hline$f\left(x_{i}\right)$ & 548159652 & 683329097 & 846302688 & 1027015247 & 1210193422 \\
\hline
\end{tabular}

Here,

$$
\begin{gathered}
x_{0}=0, x_{1}=1, x_{2}=2, x_{3}=3, x_{4}=4, \\
f\left(x_{0}\right)=548159652, \\
f\left(x_{1}\right)=683329097, \\
f\left(x_{2}\right)=846302688, \\
f\left(x_{3}\right)=1027015247 \\
\& f\left(x_{4}\right)=1210193422
\end{gathered}
$$

\begin{tabular}{|c|c|c|c|c|c|}
\hline$X$ & $f(x)$ & $\Delta f(x)$ & $\Delta^{2} f(x)$ & $\Delta^{3} f(x)$ & $\Delta^{4} f(x)$ \\
\hline 0 & 548159652 & & & & \\
\hline 1 & 683329097 & & 27804146 & & \\
\hline 2 & 846302688 & 162973591 & 17738968 & -10065178 & -5208174 \\
\hline 3 & 1027015247 & $\begin{array}{l}180712559 \\
183178175\end{array}$ & 2465616 & -15273352 & \\
\hline 4 & 1210193422 & & & & \\
\hline
\end{tabular}

\section{Finite Forward Difference Table}

Now, $C_{0}=f\left(x_{0}\right)=548159652$

$$
C_{1}=\frac{\Delta f\left(x_{0}\right)}{h}=\frac{135169445}{1}=135169445
$$




$$
\begin{aligned}
& C_{2}=\frac{\Delta^{2} f\left(x_{0}\right)}{2 ! h^{2}}=\frac{27804146}{2.1 .1}=\frac{27804146}{2}=13902073 \\
& C_{3}=\frac{\Delta^{3} f\left(x_{0}\right)}{3 ! h^{3}}=\frac{-10065178}{3.2 .1 .1}=\frac{-10065178}{6}=-1677529.66 \\
& C_{4}=\frac{\Delta^{4} f\left(x_{0}\right)}{4 ! h^{3}}=\frac{-5208174}{4.3 .2 .1 .1}=\frac{-5208174}{24}=-217007.25
\end{aligned}
$$

Accordingly,

$$
\begin{gathered}
A_{0}=548159652, A_{1}=119214356.18, A_{2}=16547582.23, \\
A_{3}=-375486.16 \& A_{4}=-217007.25
\end{gathered}
$$

Thus, the polynomial that can represent the given numerical data is

$f(x)=548159652+119214356.18 x+16547582.23 x^{2}-375486.16 x^{3}-217007.25 x^{4}$

This polynomial yields,

$$
\begin{gathered}
f(0)=548159652, \\
f(1)=683329097, \\
f(2)=846302688, \\
f(3)=1027015247 \\
\& f(4)=1210193422
\end{gathered}
$$

which are identical with the corresponding observed values.

\section{2. Formula Obtained from Newton's Backward Interpolation Formula:}

Newton's backward interpolation formula is a

$$
\begin{aligned}
f(x)= & f\left(x_{n}\right)+v \nabla f\left(x_{n-1}\right)+\frac{v(v+1)}{2 !} \nabla^{2} f\left(x_{n-2}\right)+\frac{v(v+1)(v+2)}{3 !} \nabla^{3} f\left(x_{n-3}\right)+\frac{v(v+1)(v+2))(v+3)}{4 !} \\
& \nabla^{4} f\left(x_{n-4}\right)+\ldots \ldots \ldots \ldots \ldots \ldots \ldots \ldots+\frac{v(v+1)(v+2) \ldots \ldots \ldots \ldots(v+n-1)}{n !} \quad \nabla^{n} f\left(x_{0}\right)
\end{aligned}
$$

where $v=\frac{x-x_{n}}{h}$ 
[Erdos \& Turan (1938) ; Hummel (1947) ; Jardan (1965) ; Whittaker \& Robinson (1967a) ; Kendall (1989) ; Kincard \& Ward (1991) ; Endre \& David (2003) ; Jan (2004) et al].

This formula can be expressed as

$$
\begin{aligned}
f(x)= & C_{n}+C_{n-1}\left(x-x_{n}\right)+C_{n-2}\left(x-x_{n}\right)\left(x-x_{n-1}\right)+C_{n-3}\left(x-x_{n}\right)\left(x-x_{n-1}\right)\left(x-x_{n-2}\right) \\
+ & C_{n-4}\left(x-x_{n}\right)\left(x-x_{n-1}\right)\left(x-x_{n-2}\right)\left(x-x_{n-3}\right)+\ldots \ldots \ldots \ldots \ldots \ldots \ldots \ldots \ldots \ldots \ldots \ldots \ldots \ldots \ldots \ldots \ldots \ldots \ldots \\
& \left.C_{0}\left(x-x_{n}\right)\left(x-x_{n-1}\right)\left(x-x_{n-2}\right)\left(x-x_{n-3}\right) \ldots \ldots \ldots . x_{1}\right)
\end{aligned}
$$

where $C_{n}=f\left(x_{n}\right)$

$$
\begin{gathered}
C_{n-1}=\frac{\nabla f\left(x_{n-1}\right)}{h} \\
C_{n-2}=\frac{\nabla^{2} f\left(x_{n-2}\right)}{2 ! h^{2}} \\
C_{n-3}=\frac{\nabla^{3} f\left(x_{n-3}\right)}{3 ! h^{3}} \\
C_{n-4}=\frac{\nabla^{4} f\left(x_{n-4}\right)}{4 ! h^{4}} \\
\ldots \ldots \ldots \ldots \ldots \ldots \ldots \ldots . . . \\
\ldots \ldots \ldots \ldots \ldots \ldots \ldots . . . \\
C_{0}=\frac{\nabla^{n} f\left(x_{0}\right)}{\mathrm{n} ! h^{n}}
\end{gathered}
$$

Now, equation (2.2.2), can be expressed as

$$
f(x)=A_{0}+A_{1} x+A_{2} x^{2}+A_{3} x^{3}+\ldots \ldots \ldots \ldots \ldots \ldots \ldots \ldots+A_{n} x^{n}
$$

where

$$
\begin{aligned}
& A_{0}=C_{n}-C_{n-1} x_{n}+C_{n-2} x_{n} x_{n-1}-C_{n-3} x_{n-2} x_{n-1} x_{n}+C_{n-4} x_{n-3} x_{n-2} x_{n-1} x_{n}- \\
& +(-1)^{n} C_{0}\left(x_{n} x_{n-1} x_{n-2} x_{n-3} \quad \ldots \ldots \ldots \ldots x_{1}\right) \\
& A_{1}=C_{n-1}-C_{n-2}\left(\sum_{\mathrm{i}=\mathrm{n}-1}^{\mathrm{n}} \mathrm{x}_{\mathrm{i}}\right)+C_{n-3}\left(\sum_{\mathrm{i}=\mathrm{n}-2}^{\mathrm{n}-1} \sum_{\mathrm{j}=\mathrm{n}-1}^{\mathrm{n}} \mathrm{x}_{\mathrm{i}} \mathrm{x}_{\mathrm{j}}\right)- \\
& C_{n-4}\left(\sum_{\mathrm{i}=\mathrm{n}-3}^{\mathrm{n}-2} \sum_{\mathrm{j}=\mathrm{n}-2}^{\mathrm{n}-1} \sum_{\mathrm{k}=\mathrm{n}-1}^{\mathrm{n}} \mathrm{x}_{\mathrm{i}} \mathrm{x}_{\mathrm{j}} \mathrm{x}_{\mathrm{k}}\right)+\ldots \ldots \ldots . .-C_{0}\left(\sum_{\mathrm{i}=1}^{2} \sum_{\mathrm{j}=2}^{1} \sum_{\mathrm{k}=1}^{\mathrm{n}} \mathrm{x}_{\mathrm{i}} \mathrm{x}_{\mathrm{j}} \mathrm{x}_{\mathrm{k}}\right) \\
& A_{2}=C_{n-2}-C_{n-3}\left(\sum_{\mathrm{i}=\mathrm{n}-2}^{\mathrm{n}} \mathrm{x}_{\mathrm{i}}\right)+C_{n-4}\left(\sum_{\mathrm{i}=\mathrm{n}-3}^{\mathrm{n}-1} \sum_{\mathrm{j}=\mathrm{n}-2}^{\mathrm{n}} \mathrm{x}_{\mathrm{i}} \mathrm{x}_{\mathrm{j}}\right)- \\
& +C_{0}\left(\sum_{i=2}^{3} \sum_{j=3}^{2} \sum_{k=2}^{1} \sum_{l=1}^{n} x_{i} x_{j} x_{k} x_{l}\right)
\end{aligned}
$$




$$
\begin{gathered}
A_{3}=C_{n-3}-C_{n-4}\left(\sum_{\mathrm{i}=\mathrm{n}-3}^{\mathrm{n}} \mathrm{x}_{\mathrm{i}}\right)+\ldots \ldots \ldots \ldots \ldots \ldots \ldots \ldots \ldots \ldots \ldots \ldots \ldots \ldots \ldots \ldots \ldots \ldots \ldots \ldots \ldots \ldots \\
\\
C_{0}\left(\sum_{i=3}^{4} \sum_{j=4}^{3} \sum_{k=3}^{2} \sum_{l=2}^{1} \sum_{m=1}^{n} x_{i} x_{j} x_{k} x_{l} x_{m}\right)
\end{gathered}
$$

$$
A_{n}=C_{n}
$$

Equation (2.2.3), with the coefficients

$$
A_{0}, A_{1}, A_{2}, A_{3}, \ldots \ldots \ldots \ldots \ldots \ldots \ldots, A_{n},
$$

as defined above, is another formula for representing a given set of numerical data on a pair of variables by a suitable polynomial .

Note: The formula is applicable for representing a given set of numerical data on a pair of variables by a suitable polynomial when the given values of the independent variable are at equal interval.

\section{An Example of Application of the Formula}

Example (2.2.1): Let us represent the data, given in the Example (2.1.1), by a polynomial curve applying the formula just derived.

Finite Backward Difference Table

\begin{tabular}{|c|c|c|c|c|c|}
\hline$X$ & $f(x)$ & $\nabla f(x)$ & $\nabla^{2} f(x)$ & $\nabla^{3} f(x)$ & $\nabla^{4} f(x)$ \\
\hline 0 & 548159652 & & & & \\
1 & 683329097 & 135169445 & & & \\
2 & 846302688 & 162973591 & 27804146 & & \\
3 & 1027015247 & 180712559 & 17738968 & -10065178 & -5208174 \\
4 & 1210193422 & 183178175 & 2465616 & -15273352 & \\
\hline
\end{tabular}

Here, 


$$
\begin{gathered}
C_{4}=1210193422, \mathrm{C}_{3}=183178175, C_{2}=1232808, \\
C_{1}=-2545558.66 \& C_{0}=-217007.25
\end{gathered}
$$

Consequently,

$$
\begin{gathered}
A_{0}=548159651.84, A_{1}=119214356.34, A_{2}=16547582.19, \\
A_{3}=-375486.16 \& A_{4}=-217007.25
\end{gathered}
$$

Thus the polynomial is

$f(x)=548159651.84+119214356.34 x+16547582.19 x^{2}-375486.16 x^{3}-217007.25 x^{4}$ It This This yields,

$$
\begin{gathered}
f(0)=548159652, \\
f(1)=683329097, \\
f(2)=846302688, \\
f(3)=1027015247 \\
\& f(4)=1210193422
\end{gathered}
$$

which are identical with the corresponding observed values.

\section{3. Formula Obtained from Newton's Divided Difference Interpolation Formula:}

Newton's divided difference formula is described by

$$
\begin{aligned}
& f(x)=f\left(x_{0}\right)+\left(x-x_{0}\right) f\left(x_{0}, x_{1}\right)+\left(x-x_{0}\right)\left(x-x_{1}\right) f\left(x_{0}, x_{1}, x_{2}\right)+ \\
& \left(x-x_{0}\right)\left(x-x_{1}\right)\left(x-x_{2}\right) f\left(x_{0}, x_{1}, x_{2}, x_{3}\right)+\ldots \ldots .+\left(x-x_{0}\right)\left(x-x_{1}\right)\left(x-x_{2}\right) \ldots \\
& \left(x-x_{i-1}\right) f\left(x_{0}, x_{1}, x_{2}, x_{3} \ldots . x_{i-1}\right)+\ldots \ldots \ldots . .+\left(x-x_{0}\right)\left(x-x_{1}\right)\left(x-x_{2}\right) \\
& \ldots \ldots \ldots \ldots\left(x-x_{n-1}\right) f\left(x_{0}, x_{1}, x_{2}, x_{3} \ldots \ldots x_{n}\right)
\end{aligned}
$$

where divided difference is defined by

$$
f\left(x_{0}, x_{1}, x_{2} \ldots . x_{n}\right)=\frac{f\left(x_{1}, x_{2}, x_{3} \ldots \ldots x_{n}\right)-f\left(x_{0}, x_{1}, x_{2} \ldots \ldots x_{n-1}\right)}{x_{n}-x_{0}}
$$


[Herbert (1962) ; Whittaker \& Robinson (1967a , 1967b) ; De Boor (2003) et al].

Now, divided difference interpolation formula, described by equation (2.1), can be expressed as

$$
\begin{aligned}
f(x)= & C_{0}+C_{1}\left(x-x_{0}\right)+C_{2}\left\{x^{2}-\left(\sum_{\mathrm{i}=0}^{1} \mathrm{x}_{\mathrm{i}}\right) \mathrm{x}+x_{0} x_{1}\right\}+C_{3}\left\{x^{3}-\left(\sum_{\mathrm{i}=0}^{2} \mathrm{x}_{\mathrm{i}}\right) x^{2}+\right. \\
& \left.\left(\sum_{\mathrm{i}=0}^{1} \sum_{\mathrm{j}=1}^{2} \mathrm{x}_{\mathrm{i}} \mathrm{x}_{\mathrm{j}}\right) x-x_{0} x_{1} x_{2}\right\}+C_{4}\left\{x^{4}-\left(\sum_{\mathrm{i}=0}^{3} \mathrm{x}_{\mathrm{i}}\right) x^{3}+\left(\sum_{\mathrm{i}=0}^{2} \sum_{\mathrm{j}=1}^{3} \mathrm{x}_{\mathrm{i}} \mathrm{x}_{\mathrm{j}}\right) x^{2}-\right. \\
& \left.\left(\sum_{\mathrm{i}=0}^{1} \sum_{\mathrm{j}=1}^{2} \sum_{\mathrm{k}=2}^{3} \mathrm{x}_{\mathrm{i}} \mathrm{x}_{\mathrm{j}} \mathrm{x}_{\mathrm{k}}\right) \mathrm{x}+x_{0} x_{1} x_{2} x_{3}\right\}+\ldots \ldots \ldots \ldots \ldots \ldots \ldots+C_{n}\left\{x^{n}-\left(\sum_{\mathrm{i}=0}^{\mathrm{n}-1} \mathrm{x}_{\mathrm{i}}\right) x^{n-1}\right. \\
& +\left(\sum_{\mathrm{i}=0}^{\mathrm{n}-2} \sum_{\mathrm{j}=1}^{\mathrm{n}-1} \mathrm{x}_{\mathrm{i}} \mathrm{x}_{\mathrm{j}}\right) x^{n-2}-\left(\sum_{\mathrm{i}=0}^{\mathrm{n}-3} \sum_{\mathrm{j}=1}^{\mathrm{n}-2} \sum_{\mathrm{k}=2}^{\mathrm{n}-1} \mathrm{x}_{\mathrm{i}} \mathrm{x}_{\mathrm{j}} \mathrm{x}_{\mathrm{k}}\right) x^{n-3}+ \\
& \left.\left(\sum_{\mathrm{i}=0}^{\mathrm{n}-3} \sum_{\mathrm{j}=1}^{\mathrm{n}-2} \sum_{\mathrm{k}=2}^{\mathrm{n}-1} \sum_{\mathrm{l}=3}^{\mathrm{n}} \mathrm{x}_{\mathrm{i}} \mathrm{x}_{\mathrm{j}} \mathrm{x}_{\mathrm{k}} \mathrm{x}_{\mathrm{l}}\right) x^{n-4}+\ldots \ldots \ldots+(-1)^{n}\left(x_{0} x_{1} x_{2} x_{3} \ldots \ldots x_{n-1}\right)\right\}
\end{aligned}
$$

where

$$
C_{i}=f\left(x_{0}, x_{1}, x_{2}, x_{3} \ldots \ldots x_{i}\right), i=1,2,3, \ldots \ldots \ldots \ldots \ldots \ldots,(n-1), n
$$

Now, equation (2.3.2), can be expressed as

$$
f(x)=A_{0}+A_{1} x+A_{2} x^{2}+A_{3} x^{3}+\ldots \ldots \ldots \ldots \ldots \ldots \ldots \ldots+A_{n} x^{n}
$$

where

$$
\begin{aligned}
& A_{0}=C_{0}-C_{1} x_{0}+C_{2} x_{0} x_{1}-C_{3} x_{0} x_{1} x_{2}+C_{4} x_{0} x_{1} x_{2} x_{3}- \\
& +C_{n}(-1)^{n}\left(x_{0} x_{1} x_{2} x_{3} \ldots \ldots \ldots \ldots x_{n-1}\right) \\
& A_{1}=C_{1}-C_{2}\left(\sum_{\mathrm{i}=0}^{1} \mathrm{x}_{\mathrm{i}}\right)+C_{3}\left(\sum_{\mathrm{i}=0}^{1} \sum_{\mathrm{j}=1}^{2} \mathrm{x}_{\mathrm{i}} \mathrm{x}_{\mathrm{j}}\right)-C_{4}\left(\sum_{\mathrm{i}=0}^{1} \sum_{\mathrm{j}=1}^{2} \sum_{\mathrm{k}=2}^{3} \mathrm{x}_{\mathrm{i}} \mathrm{x}_{\mathrm{j}} \mathrm{x}_{\mathrm{k}}\right)+ \\
& +(-1)^{n} C_{n}\left(x_{0} x_{1} x_{2} x_{3} \ldots \ldots \ldots \ldots x_{n-2}+x_{0} x_{1} x_{2} x_{3} \ldots \ldots \ldots \ldots x_{n-1}\right) \\
& A_{2}=C_{2}-C_{3}\left(\sum_{\mathrm{i}=0}^{2} \mathrm{x}_{\mathrm{i}}\right)+C_{4}\left(\sum_{\mathrm{i}=0}^{2} \sum_{\mathrm{j}=1}^{3} \mathrm{x}_{\mathrm{i}} \mathrm{x}_{\mathrm{j}}\right)- \\
& (-1)^{n} C_{n}\left(x_{0} x_{1} x_{2} x_{3} \ldots \ldots \ldots \ldots x_{n-3}+x_{0} x_{1} x_{2} x_{3} \ldots \ldots \ldots \ldots x_{n-2}+\right. \\
& \left.x_{0} x_{1} x_{2} x_{3} \ldots \ldots \ldots \ldots x_{n-1}\right) \\
& A_{3}=C_{3}-C_{4}\left(\sum_{\mathrm{i}=0}^{3} \mathrm{x}_{\mathrm{i}}\right)+\ldots \ldots \ldots . .+(-1)^{n} C_{n}\left(x_{0} x_{1} x_{2} x_{3} \ldots \ldots \ldots \ldots x_{n-4}+\right. \\
& \left.x_{0} x_{1} x_{2} x_{3} \ldots \ldots \ldots x_{n-3}+x_{0} x_{1} x_{2} x_{3} \ldots \ldots \ldots x_{n-2}+x_{0} x_{1} x_{2} x_{3} \ldots \ldots \ldots x_{n-1}\right) \\
& A_{i}=C_{i}-C_{i+1}\left(\sum_{\mathrm{j}=0}^{\mathrm{i}} \mathrm{x}_{\mathrm{i}}\right)+\ldots \ldots \ldots \ldots \ldots \ldots+(-1)^{n-i} C_{n}\left(x_{0} x_{1} x_{2} x_{3} \ldots \ldots \ldots \ldots x_{n-i}\right. \\
& \left.+\ldots \ldots \ldots \ldots \ldots \ldots \ldots \ldots+x_{0} x_{1} x_{2} x_{3} \ldots \ldots \ldots \ldots x_{n-1}\right)
\end{aligned}
$$




$$
A_{n}=C_{n}
$$

Equation (2.3.3), with the coefficients

$$
A_{0}, A_{1}, A_{2}, A_{3}, \ldots \ldots \ldots \ldots \ldots \ldots \ldots, A_{n}
$$

as defined above, is another formula for representing a given set of numerical data on a pair of variables by a suitable polynomial .

Note: For the applicability of the formula for representing a set of numerical data on a pair of variables by a suitable polynomial it is not necessary that the values of the independent variable should be at equal interval.

\section{An Example of Application of the Formula}

Example (2.3.1): Let us represent the data, given in the Example (2.1.1), by a polynomial curve applying the formula just derived.

\section{Divided Difference Table}

\begin{tabular}{|c|c|c|c|c|c|}
\hline$x$ & $f(x)$ & $f\left(x_{0}, x_{1}\right)$ & $f\left(x_{0}, x_{1}, x_{2}\right)$ & $f\left(x_{0}, x_{1}, x_{2}, x_{3}\right)$ & $f\left(x_{0}, x_{1}, x_{2}, x_{3}, x_{4}\right)$ \\
\hline 0 & 548159652 & & & & \\
1 & 683329097 & 135169445 & & & \\
2 & 846302688 & 162973591 & 8869484 & -1677529.66 & -217007.25 \\
3 & 1027015247 & 180712559 & 1232808 & & \\
4 & 1210193422 & & & & \\
\hline
\end{tabular}

Here,

$$
\begin{gathered}
C_{0}=548159652, C_{1}=135169445, C_{2}=13902073, \\
C_{3}=-1677529.66 \& C_{4}=-217007.25
\end{gathered}
$$


Accordingly,

$$
\begin{gathered}
A_{0}=548159652, A_{1}=119214356.18, A_{2}=16547582.23, \\
A_{3}=-375486.16 \& A_{4}=-217007.25
\end{gathered}
$$

Thus, the polynomial that can represent the given numerical data is $f(x)=548159652+119214356.18 x+16547582.23 x^{2}-375486.16 x^{3}-217007.25 x^{4}$ This polynomial yields,

$$
\begin{gathered}
f(0)=548159652, \\
f(1)=683329097, \\
f(2)=846302688, \\
f(3)=1027015247 \\
\& f(4)=1210193422
\end{gathered}
$$

which are also identical with the corresponding observed values.

\section{4. Formula Obtained from Lagrange's Interpolation Formula:}

Lagrange's interpolation formula is described by

$$
\begin{aligned}
& f(x)=\frac{\left(x-x_{1}\right)\left(x-x_{2}\right)\left(x-x_{3}\right) \ldots \ldots \ldots \ldots . .\left(x-x_{n}\right)}{\left(x_{0}-x_{1}\right)\left(x_{0}-x_{1}\right) \ldots \ldots \ldots\left(x_{0}-x_{1}\right)} y_{0}+\frac{\left(x-x_{0}\right)\left(x-x_{2}\right)\left(x-x_{3}\right) \ldots \ldots \ldots \ldots\left(x-x_{n}\right)}{\left(x_{1}-x_{0}\right)\left(x_{1}-x_{2}\right)\left(x_{1}-x_{3}\right) \ldots \ldots \ldots\left(x_{1}-x_{n}\right)} \quad y_{1}
\end{aligned}
$$

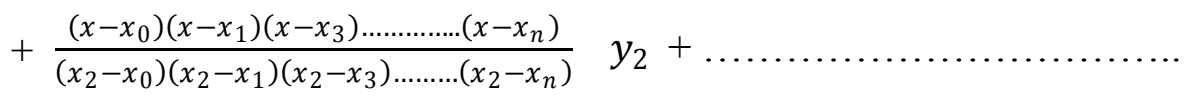

$$
\begin{aligned}
& +\frac{\left(x-x_{0}\right)\left(x-x_{1}\right)\left(x-x_{2}\right) \ldots \ldots \ldots \ldots .\left(x-x_{n-1}\right)}{\left(x_{n}-x_{0}\right)\left(x_{n}-x_{1}\right)\left(x_{n}-x_{2}\right) \ldots \ldots . .\left(x_{n}-x_{n-1}\right)} \quad y_{n}
\end{aligned}
$$

[Echols (1893) ; Corliss (1938) ; Hummel (1947) ; Traub (1964) ; Jardan (1965) ; Quadling (1966) ; Mills (1977) ; Kendall (1989); Kincard \& Ward (1991) ; Revers \& Michael (2000) ; Endre \& David (2003) ; Jan (2004) ; Nasrin (2010) et al].

Now, Lagranges interpolation formula, described by equation (2.1), can be expressed as

$$
\begin{aligned}
& f(x)=\frac{\prod_{\mathrm{i}=0, \mathrm{i} \neq 0}^{\mathrm{n}}\left(\mathrm{x}-\mathrm{x}_{\mathrm{i}}\right)}{\prod_{\mathrm{i}=0, \mathrm{i} \neq 0}^{\mathrm{n}}\left(\mathrm{x}_{0}-\mathrm{x}_{\mathrm{i}}\right)} f\left(x_{0}\right)+\frac{\prod_{\mathrm{i}=0, \mathrm{i} \neq 1}^{\mathrm{n}}\left(\mathrm{x}-\mathrm{x}_{\mathrm{i}}\right)}{\prod_{\mathrm{i}=0, \mathrm{i} \neq 1}\left(\mathrm{x}_{1}-\mathrm{x}_{\mathrm{i}}\right)} f\left(x_{l}\right)+ \\
& +\ldots \ldots \ldots \ldots \ldots \ldots+\cdots+\frac{\prod_{i=0, i \neq n-1}^{n-1}\left(x-x_{i}\right)}{\prod_{i=0, i \neq n-1}^{n-1}\left(x_{n}-x_{i}\right)} f\left(x_{n}\right) \\
& =\sum_{\mathbf{r}=\mathbf{0}}^{\mathbf{n}}\left\{\frac{\mathrm{f}\left(\mathrm{x}_{\mathrm{r}}\right)}{\prod_{\mathrm{i}=0, \mathrm{i} \neq \mathrm{r}}^{\mathrm{r}}\left(\mathrm{x}_{0}-\mathrm{x}_{\mathrm{i}}\right)}\right\} \prod_{\mathrm{i}=0, \mathrm{i} \neq \mathrm{r}}^{\mathrm{r}}\left(\mathrm{x}-\mathrm{x}_{\mathrm{i}}\right)
\end{aligned}
$$


The expression (2.4.2) can be written as

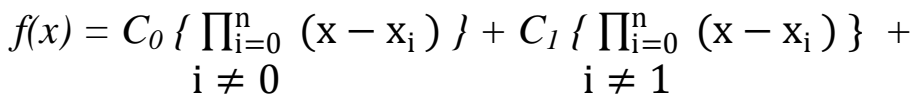

$$
\begin{aligned}
& C_{2}\left\{\prod_{\mathrm{i}=0}^{\mathrm{n}}\left(\mathrm{x}-\mathrm{x}_{\mathrm{i}}\right)\right\}+\ldots \ldots \ldots \ldots+C_{r}\left\{\prod_{\mathrm{i}=0}^{\mathrm{n}}\left(\mathrm{x}-\mathrm{x}_{\mathrm{i}}\right)\right\}+\ldots \ldots \ldots \ldots \ldots \ldots \\
& +C_{n}\left\{\prod_{\substack{\mathrm{n}=0 \\
i \neq \mathrm{n}}}^{\mathrm{n}}\left(\mathrm{x}-\mathrm{x}_{\mathrm{i}}\right)\right\}
\end{aligned}
$$

where $\quad C_{i}=\frac{\mathrm{f}\left(x_{1}\right)}{\prod_{\mathrm{i}=0}^{\mathrm{n}}\left(\mathrm{x}_{\mathrm{j}}-\mathrm{x}_{\mathrm{i}}\right)}$

This can further be expressed as

$$
\begin{aligned}
& f(x)=\left(\sum_{\mathrm{i}=0}^{\mathrm{n}} \mathrm{C}_{\mathrm{i}}\right) \mathrm{x}^{\mathrm{n}}+\left\{\sum_{\mathrm{i}=0}^{\mathrm{n}} \mathrm{C}_{\mathrm{i}} \mathrm{S}_{\mathrm{i}}(1)\right\} \mathrm{x}^{\mathrm{n}-1}+\left\{\sum_{\mathrm{i}=0}^{\mathrm{n}} \mathrm{C}_{\mathrm{i}} \mathrm{S}_{\mathrm{i}}(2)\right\} \mathrm{x}^{\mathrm{n}-2}+ \\
&\left\{\sum_{\mathrm{i}=0}^{\mathrm{n}} \mathrm{C}_{\mathrm{i}} \mathrm{S}_{\mathrm{i}}(3)\right\} \mathrm{x}^{\mathrm{n}-3}+\ldots \ldots \ldots \ldots \ldots \ldots \ldots \ldots \ldots \ldots \ldots \ldots \ldots \ldots \ldots \ldots \ldots \ldots \ldots \\
&+\left\{\sum_{\mathrm{i}=0}^{\mathrm{n}} \mathrm{C}_{\mathrm{i}} \mathrm{S}_{\mathrm{i}}(\mathrm{r})\right\} \mathrm{x}^{\mathrm{n}-\mathrm{r}} \\
&\left.\mathrm{S}_{\mathrm{i}}(\mathrm{n}-\mathrm{r})\right\} \mathrm{x}+\sum_{\mathrm{i}=0}^{\mathrm{n}} \mathrm{C}_{\mathrm{i}} \mathrm{S}_{\mathrm{i}}(n)
\end{aligned}
$$

where $\quad S_{r}=\sum_{\mathrm{i}=0}^{\mathrm{n}} \mathrm{x}_{\mathrm{i}} \quad, \quad S_{r}(p)=\sum_{\mathrm{i}=0}^{\mathrm{n}} \sum_{\mathrm{j}=0}^{\mathrm{n}} \mathrm{x}_{\mathrm{i}} \mathrm{x}_{\mathrm{j}}$

$i \neq r \quad i \neq r, j \neq r, i \neq j$

$$
\begin{gathered}
\mathrm{S}_{\mathrm{r}_{1} \mathrm{r}_{2} \ldots \ldots \ldots . . \mathrm{r}_{\mathrm{p}}}=\sum_{\mathrm{i}_{1}=0}^{\mathrm{n}} \sum_{\mathrm{i}_{2}=0}^{\mathrm{n}} \ldots \ldots \ldots \sum_{\mathrm{i}_{\mathrm{p}}=0}^{\mathrm{n}} \mathrm{x}_{\mathrm{i}_{1}} \mathrm{x}_{\mathrm{i}_{2}} \ldots \ldots \mathrm{x}_{\mathrm{i}_{\mathrm{p}}} \\
\mathrm{i}_{1} \neq \mathrm{r}_{1}, \mathrm{i}_{2} \neq \mathrm{r}_{1} \ldots \ldots \ldots \mathrm{i}_{\mathrm{p}} \neq \mathrm{r}_{\mathrm{p}} \\
\mathrm{i}_{1} \neq \mathrm{i}_{2} \neq----\neq \mathrm{i}_{\mathrm{p}}
\end{gathered}
$$

This is the formula for representation of numerical data by a polynomial curve obtained from Lagrange's interpolation formula.

\section{An Example of Application of the Formula}

Example (2.4.1): Let us represent the data, given in the Example (2.1.1), by a polynomial curve applying the formula just derived.

Here,

$$
C_{0}=22839985.5, C_{1}=-113888182.83, C_{2}=211575672,
$$




$$
C_{3}=-171169207.83 \& C_{4}=50424725.91
$$

$$
\begin{aligned}
& \therefore C_{0}+C_{1}+C_{2}+C_{3}+C_{4} \\
& =-217007.25
\end{aligned}
$$

Also,

$$
\begin{aligned}
& C_{0}\left(x_{1}+x_{2}+x_{3}+x_{4}\right)+C_{1}\left(x_{0}+x_{2}+x_{3}+x_{4}\right)+C_{2}\left(x_{0}+x_{1}+x_{3}+x_{4}\right)+C_{3}\left(x_{0}+x_{1}+x_{2}+x_{4}\right) \\
& \quad+C_{4}\left(x_{0}+x_{1}+x_{2}+x_{3}\right) \\
& \quad 375486.2
\end{aligned}
$$

Again ,

$$
\begin{aligned}
& C_{0}\left(x_{1} x_{2}+x_{1} x_{3}+x_{1} x_{4}+x_{2} x_{3}+x_{2} x_{4}+x_{3} x_{4}\right)+C_{1}\left(x_{0} x_{2}+x_{0} x_{3}+x_{0} x_{4}+x_{2} x_{3}+x_{2} x_{4}+\right. \\
& \left.x_{3} x_{4}\right)+C_{2}\left(x_{0} x_{1}+x_{0} x_{3}+x_{0} x_{4}+x_{1} x_{3}+x_{1} x_{4}+x_{3} x_{4}\right)+C_{3}\left(x_{0} x_{1}+x_{0} x_{2}+x_{0} x_{4}+\right. \\
& \left.x_{1} x_{2}+x_{1} x_{4}+x_{2} x_{4}\right)+C_{4}\left(x_{0} x_{1}+x_{0} x_{2}+x_{0} x_{3}+x_{1} x_{2}+x_{1} x_{3}+x_{2} x_{3}\right) \\
= & 16547582.3, \\
& C_{0}\left(x_{1} x_{2} x_{3}+x_{1} x_{3} x_{4}+x_{2} x_{3} x_{4}+x_{1} x_{2} x_{4}\right)+C_{1}\left(x_{0} x_{2} x_{3}+x_{0} x_{2} x_{4}+x_{0} x_{3} x_{4}+x_{2} x_{3} x_{4}\right) \\
+ & \mathrm{C}_{2}\left(x_{0} x_{1} x_{3}+x_{0} x_{3} x_{4}+x_{0} x_{1} x_{4}+x_{1} x_{3} x_{4}\right)+\mathrm{C}_{3}\left(x_{0} x_{1} x_{2}+x_{0} x_{2} x_{4}+x_{0} x_{1} x_{4}+x_{1} x_{2} x_{4}\right) \\
& \mathrm{C}_{4}\left(x_{0} x_{1} x_{2}+x_{0} x_{1} x_{3}+x_{0} x_{2} x_{3}+x_{1} x_{2} x_{3}\right) \\
= & -119214356, \\
\& & C_{0}\left(x_{1} x_{2} x_{3} x_{4}\right)+C_{1}\left(x_{0} x_{2} x_{3} x_{4}\right)+C_{2}\left(x_{0} x_{1} x_{3} x_{4}\right)+C_{3}\left(x_{0} x_{1} x_{2} x_{4}\right)+C_{4}\left(x_{0} x_{1} x_{2} x_{3}\right) \\
= & 548159652
\end{aligned}
$$

Therefore, the polynomial that can represent the given numerical data is

$$
f(x)=-217007.25 x^{4}-375486.2 x^{3}+16547582.3 x^{2}+119214356 x+548159652
$$

This polynomial yields,

$$
\begin{gathered}
f(0)=548159652, f(1)=683329097, f(2)=846302688, \\
f(3)=1027015246.1 \& f(4)=1210193432
\end{gathered}
$$

which are also identical with the corresponding observed values. 


\section{5. Method Based on Difference and Ratio Operators:}

The calculus of finite difference [Gertrude(1954); Herbert (1962); Jordan (1965); Dokken \& Lyche (1979) ; Fred (1979) ; Jeffreys \& Jeffreys (1988) ; Lee (1989) ; Chwaiger (1994) ; De Boor (2003) ; Endre \& David (2003) ; Floater (2003) et al] has been applied in developing this method.

The $(n+1)$ pairs of values namely

$$
\left(x_{0}, y_{0}\right),\left(x_{1}, y_{1}\right),\left(x_{2}, y_{2}\right), \ldots \ldots \ldots \ldots,\left(x_{n-1}, y_{n-1}\right),\left(x_{n}, y_{n}\right)
$$

are nothing but $(n+1)$ points in $X-Y$ plane

The objective is to represent these $n$ points by a polynomial of the form

$$
y=f(x)=a_{0}+a_{1} x+a_{2} x^{2}+a_{3} x^{3} \ldots \ldots \ldots \ldots \ldots+a_{n} x^{n}, \quad a_{n} \neq 0
$$

where

$a_{0}, a_{1}, a_{2}, a_{3}, \ldots \ldots \ldots \ldots, a_{n}$ are parameters.

In this study, two operators namely difference operator (denoted by $\Delta$ ) and radio operator (denoted by $R[$. : . . have been used in the derivation of the method aimed at.

The operator $\Delta$ has been defined with respect to the suffix variable as

$$
\Delta x_{i}=x_{i+1}-x_{i} \quad \& \quad \Delta y_{i}=y_{i+1}-y_{i}
$$

while the operator $R[$. : . . has been defined as

$$
R[f(.): h(.)]=\frac{f(.)}{h(.)}
$$

i.e. $R[f():. g()$.$] denotes the ratio of g() \& f.($.$) .$

$$
y=f(x)=a_{0}+a_{1} x+a_{2} x^{2}+\ldots \ldots \ldots \ldots \ldots+a_{n} x^{n}, \quad a_{n} \neq 0
$$

Since the $(n+1)$ points pass through the curve

therefore,

$$
y_{i}=a_{0}+a_{1} x_{i}+a_{2} x_{i}^{2}+a_{3} x_{i}^{3}+\ldots \ldots \ldots \ldots \ldots+a_{n} x_{i}^{n},
$$

$(i=0,1,2,3$, ,n).

This yield 


$$
\begin{aligned}
\Delta y_{i} & =a_{1} \Delta x_{i}+a_{2} \Delta x_{i}^{2}+a_{3} \Delta x_{i}^{3}+\ldots \ldots \ldots \ldots \ldots+a_{n} \Delta x_{i}^{n} \\
\text { i.e. } y_{i}(1) & =a_{1}+a_{2} x_{i}^{2}(1)+a_{3} x_{i}^{3}(1)+\ldots \ldots \ldots \ldots .+a_{n} x_{i}^{n}(1)
\end{aligned}
$$

where

$$
\begin{gathered}
y_{i}(1)=R\left[\Delta y_{i}: \Delta x_{i}\right], \\
x_{i}^{2}(1)=R\left[\Delta x_{i}^{2}: \Delta x_{i}\right], \\
x_{i}^{3}(1)=R\left[\Delta x_{i}^{3}: \Delta x_{i}\right], \\
\ldots \ldots \ldots \ldots \ldots \ldots \ldots, \\
x_{i}^{n}(1)=R\left[\Delta x_{i}^{n}: \Delta x_{i}\right], \\
(i=0,1,2,3, \ldots \ldots \ldots \ldots, n-1) .
\end{gathered}
$$

This yield,

$$
\begin{aligned}
\Delta y_{i}(1) & =a_{2} \Delta x_{i}{ }^{2}(1)+a_{3} \Delta x_{i}{ }^{3}(1)+\ldots \ldots \ldots \ldots .+a_{n} \Delta x_{i}{ }^{n}(1) \\
\text { i.e. } y_{i}(2) & =a_{2}+a_{3} x_{i}{ }^{3}(2)+\ldots \ldots \ldots \ldots .+a_{n} x_{i}{ }^{n}(2)
\end{aligned}
$$

where

$$
\begin{gathered}
y_{i}(2)=R\left[\Delta y_{i}(1): \Delta x_{i}^{2}(1)\right], \\
x_{i}{ }^{3}(2)=R\left[\Delta x_{i}^{3}(1): \Delta x_{i}^{2}(1)\right], \\
\ldots \ldots \ldots \ldots \ldots \ldots \ldots \ldots, \\
x_{i}^{n}(2)=R\left[\Delta x_{i}^{n}(1): \Delta x_{i}^{2}(1)\right] \\
(i=0,1,2,3, \ldots \ldots \ldots \ldots, n-2) .
\end{gathered}
$$

This yield

$$
\Delta y_{i}(2)=a_{3} \Delta x_{i}^{3}(2)+\ldots \ldots \ldots \ldots . .+a_{n} \Delta x_{i}^{n}(2)
$$

$$
\text { i.e. } y_{i}(3)=a_{3}+\ldots \ldots \ldots \ldots .+a_{n} x_{i}{ }^{n}(3)
$$


where

$$
\begin{gathered}
y_{i}(3)=R\left[\Delta y_{i}(2): \Delta x_{i}{ }^{3}(2)\right], \\
x_{i}^{4}(3)=R\left[\Delta x_{i}^{4}(2): \Delta x_{i}^{3}(2)\right], \\
\ldots \ldots \ldots \ldots \ldots \ldots \ldots ., \\
x_{i}^{n}(3)=R\left[\Delta x_{i}^{n}(2): \Delta x_{i}^{3}(2)\right] \\
(i=0,1,2,3, \ldots \ldots \ldots \ldots, n-3) .
\end{gathered}
$$

At the $k^{\text {th }}$ step, one can obtain that

$$
\begin{aligned}
& \Delta y_{i}(k-1)=a_{k} \Delta x_{i}^{k}(k-1)+a_{k+1} \Delta x_{i}^{k+1}(k-1)+\ldots \ldots \ldots \ldots \ldots+a_{n} \Delta x_{i}^{n}(k-1) \\
& \text { i.e. } y_{i}(k)=a_{k}+a_{k+1} \Delta x_{i}{ }^{k+1}(k)+\ldots \ldots \ldots \ldots \ldots+a_{n} \Delta x_{i}{ }^{n}(k)
\end{aligned}
$$

where

$$
\begin{gathered}
y_{i}(k)=R\left[\Delta y_{i}(k-1): \Delta x_{i}{ }^{k}(k-1)\right], \\
\left.x_{i}{ }^{k+1}(k)=R \Delta x_{i}{ }^{k+1}(k-1): \Delta x_{i}{ }^{k}(k-1)\right], \\
\ldots \ldots \ldots \ldots \ldots \ldots \ldots \ldots \ldots \ldots \ldots \ldots \ldots . . . \\
\Delta x_{i}{ }^{n}(k)=R\left[\Delta x_{i}{ }^{n}(k-1): \Delta x_{i}{ }^{k}(k-1)\right], \\
(i=0,1,2,3, \ldots \ldots \ldots \ldots .
\end{gathered}
$$

Continuing the process, one can arrive at the $(n-1)^{\text {th }}$ step that

$$
\begin{aligned}
\Delta y_{i}(n-2) & =a_{n-1} \Delta x_{i}^{n-1}(n-2)+a_{n} \Delta x_{i}^{n}(n-2) \\
\text { i.e. } y_{i}(n-1) & =a_{n-1}+a_{n} x_{i}{ }^{n}(n-1)
\end{aligned}
$$

where

$$
y_{i}(n-1)=R\left[\Delta y_{i}(n-2): \Delta x_{i}^{n-1}(n-2)\right]
$$

$(i=0,1)$. 
At the $n^{\text {th }}$ step, one obtain that

$$
\Delta y_{0}(n-1)=a_{n} \Delta x_{0}^{n}(n-1)
$$

i.e. $a_{n}=y_{0}(n)$ where $y_{0}(n)=R\left[\Delta y_{0}(n-1): \Delta x_{0}{ }^{n}(n-1)\right]$

Substituting the value of $a_{n}$ in any of the two equations given by (2.37) and then solving it one can obtain the value of $a_{n-1}$.

Next, substituting the values of $a_{n} \quad \& a_{n-1}$ in any of the three equations, containing $a_{n}, a_{n-1} \& a_{n-2}$, obtained in the just earlier steps one can obtain the value of $a_{n-2}$. Similarly in the next step, substituting the values of $a_{n}, a_{n-1} \& a_{n-2}$ in any of the four equations, containing $a_{n}, a_{n-1}, a_{n-2} \& a_{n-3}$ obtained in the just earlier steps one can obtain the value of $a_{n-3}$.

Continuing the process one can obtain the values of the parameters in the order

$$
a_{n}, a_{n-1}, a_{n-2}, a_{n-3}, \ldots \ldots \ldots \ldots \ldots, a_{2}, a_{1}, a_{0} .
$$

\section{An Example of Application of the Method}

Example (2.5.1): Let us represent the data, given in the Example (2.1.1), by a polynomial curve applying the formula just derived.

It is wanted to represent these data by a polynomial curve of degree four defined by equation (2.22).

In this case,

$$
\begin{gathered}
n=4, x_{0}=0, x_{1}=1, x_{2}=2, x_{3}=3, x_{4}=4, \\
y_{0}=f\left(x_{0}\right)=548159652, y_{1}=f\left(x_{1}\right)=683329097, \\
f\left(x_{2}\right)=846302688, f\left(x_{3}\right)=1027015247 \quad \& \quad f\left(x_{4}\right)=1210193422 .
\end{gathered}
$$

Applying the necessary steps in the proper order it is found that

$$
\begin{aligned}
& e=-217007.25 \\
& d=-375486.2, \\
& c=16547582.37
\end{aligned}
$$




$$
\begin{gathered}
b=119214356 \\
\& a=548159652 .
\end{gathered}
$$

Hence, the equation of the polynomial curve of degree four that can represent the given numerical data is

$$
y=f(x)=-217007.25 x^{4}-375486.2 x^{3}+16547582.3 x^{2}+119214356 x+548159652
$$

The values of the function $y=f(x)$ corresponding to the obse4rved values of $x$ yielded by this polynomial curve are as follows:

$$
\begin{gathered}
f(0)=548159652, \\
f(1)=683329097, \\
f(2)=846302687.6, \\
f(3)=1027015246.1 \\
\& f(4)=1210193432 .
\end{gathered}
$$

These values are found to be identical with the corresponding observed values.

\section{6. Method Based on Matrix Inversion by Cayley-Hamilton Theorem:}

Since the $(n+1)$ points

$$
\left(x_{0}, y_{0}\right),\left(x_{1}, y_{1}\right),\left(x_{2}, y_{2}\right), \ldots \ldots \ldots \ldots,\left(x_{n-1}, y_{n-1}\right),\left(x_{n}, y_{n}\right)
$$

lie on the polynomial curve described by

$$
y=f(x)=a_{0}+a_{1} x+a_{2} x^{2}+a_{3} x^{3} \ldots \ldots \ldots \ldots \ldots+a_{n} x^{n}, \quad a_{n} \neq 0
$$

therefore,

$$
y_{i}=f\left(x_{i}\right)=a_{0}+a_{1} x_{i}+a_{2} x_{i}^{2}+a_{2} x_{i}^{3}+\ldots \ldots \ldots+a_{2} x_{i}^{n}
$$

In order to obtain the polynomial we are to solve this n equations for the $n$ unknown coefficients $a_{0}, a_{1}, \ldots \ldots \ldots \ldots, a_{n}$.

Now solving these equations for the coefficients, the polynomial can be obtained. 
In order to solve the equations it is to be noted that the equations in (2.1) can be expressed as

$$
A X=B
$$

where

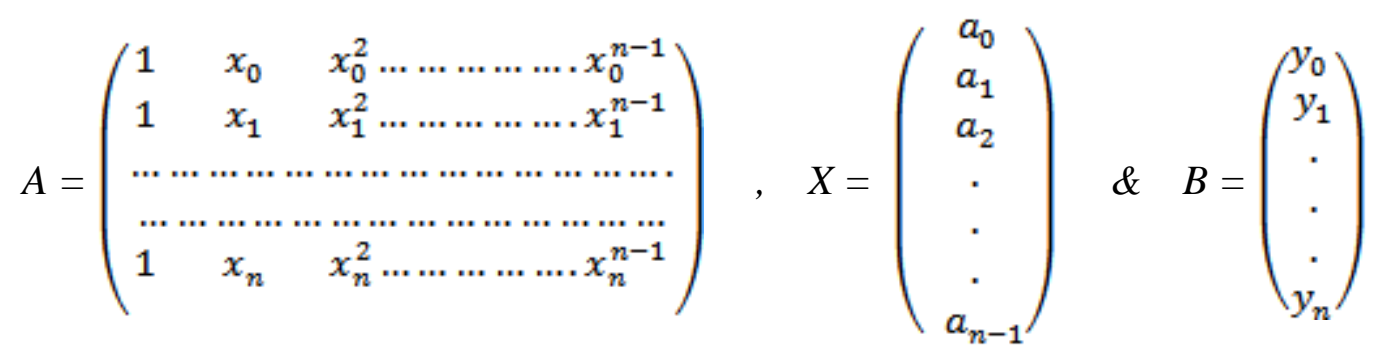

which gives

$$
X=A^{-1} B
$$

Thus in order to find out $X$, it is required to find out $A^{-1}$.

Now, $\left|\mathrm{A}_{n}-\lambda \mathrm{I}_{n}\right|$ is the characteristic polynomial and

$$
\left|\mathrm{A}_{n}-\lambda \mathrm{I}_{n}\right|=0
$$

is the characteristic equation of the matrix $A$.

Let the characteristic equation of $A$ be

$$
\lambda^{n}+c_{1} \lambda^{n}+c_{2} \lambda^{n-}+c_{3} \lambda^{n-}+\ldots \ldots \ldots \ldots \ldots \ldots \ldots \ldots+c_{n}=0
$$

By Caley-Hamilton theorem,

$$
\mathrm{A}^{n}+c_{1} \mathrm{~A}^{n-1}+c_{2} \mathrm{~A}^{n-2}+c_{3} \mathrm{~A}^{n-3}+\ldots \ldots \ldots \ldots \ldots \ldots \ldots \ldots+c_{n} I_{n}=0
$$

[Cayley $(1858,1889)$; Hamilton $(1853,1862,1864 a, 1864 b)$ et al].

Therefore,

$$
A^{-1}=-\frac{1}{v_{n}}\left[\mathrm{~A}^{n-1}+c_{1} \mathrm{~A}^{n-2}+c_{2} \mathrm{~A}^{n-3}+c_{3} \mathrm{~A}^{n-4}+\ldots \ldots \ldots+c_{n-1} I_{n}\right]
$$

Thus in order to find out $A^{-1}$ it is required to find out the characteristic polynomial and the characteristic equation of $A$.

By algebraic expansion, the characteristic polynomial of the matrix 


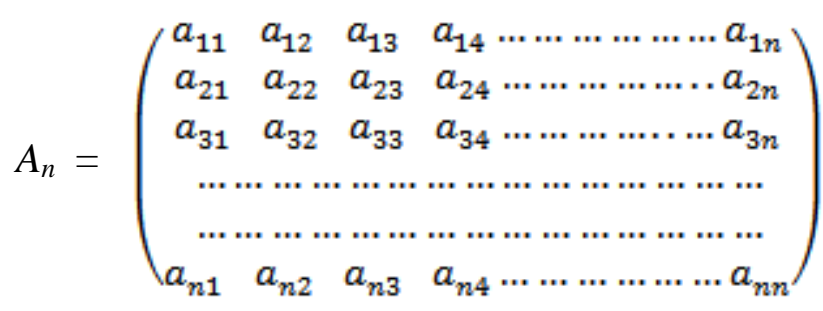

can be obtained as

$$
\begin{gathered}
|\mathrm{A}-\lambda \mathrm{I}|==(-1)^{n} \lambda^{n}+(-1)^{n-1}\left(\sum_{i=1}^{4} 1\right) \lambda^{n-1}+(-1)^{n-2}\left\{\sum \sum_{i<j=1}^{n} 1 . x_{1}-\right. \\
\left.\sum \sum_{i<j=1}^{n} x_{i-1}^{j-1} x_{j-1}^{i-1}\right\} \lambda^{n-2}+(-1)^{n-3}\left\{\sum \sum \sum_{i<j<k=1}^{n} x_{i-1}^{j-1} \cdot x_{1} x_{2}^{2}-\sum_{i=1}^{n} 1\left(\sum_{j=1}^{n} \sum_{k=1}^{n} x_{j-1}^{k-1} x_{k-1}^{j-1}\right)\right. \\
\mathrm{j} \neq \mathrm{k}
\end{gathered}
$$

\section{An Example of Application of the Formula:}

The following table shows the data on total population of India corresponding to the years:

\begin{tabular}{|c|c|c|c|c|}
\hline Year & 1971 & 1981 & 1991 & 2001 \\
\hline $\begin{array}{c}\text { Total } \\
\text { Population }\end{array}$ & 548159652 & 683329097 & 846302688 & 1027015247 \\
\hline
\end{tabular}

Taking 1971 as origin and changing scale by 1/10, one can obtain the following table for independent variable $x$ (representing time) and $f(x)$ (representing total population of India): 


\begin{tabular}{|c|c|c|c|c|}
\hline Year & 1971 & 1981 & 1991 & 2001 \\
\hline$x_{i}$ & 0 & 1 & 2 & 3 \\
\hline$f\left(x_{i}\right)$ & 548159652 & 683329097 & 846302688 & 1027015247 \\
\hline
\end{tabular}

Here,

$$
\begin{gathered}
x_{0}=0, x_{1}=1, x_{2}=2, x_{3}=3, \\
f\left(x_{0}\right)=548159652, f\left(x_{1}\right)=683329097, \\
f\left(x_{2}\right)=846302688 \& f\left(x_{3}\right)=1027015247,
\end{gathered}
$$

Thus,

$$
\mathrm{X}=\left(\begin{array}{l}
a_{0} \\
a_{1} \\
a_{2} \\
a_{3}
\end{array}\right), \quad \mathrm{A}=\left(\begin{array}{cccc}
1 & 0 & 0 & 0 \\
1 & 1 & 1 & 1 \\
1 & 2 & 4 & 8 \\
1 & 3 & 9 & 27
\end{array}\right), \quad \mathrm{B}=\left(\begin{array}{l}
y_{0} \\
y_{1} \\
y_{2} \\
y_{3}
\end{array}\right)
$$

$|A-\lambda I|=\lambda-33 \lambda+94 \lambda-74 \lambda+12$

$\therefore$ The characteristic equation is

$$
\lambda-33 \lambda+94 \lambda-74 \lambda+12=0
$$

By Caley Hamilton theorem, we have

$$
\begin{aligned}
& A^{4}-33 A^{3}+94 A^{2}-74 A+12 I=0 \\
\Rightarrow & 12 I=-A^{4}+33 A^{3}-94 A^{2}+74 A \\
\Rightarrow & I=\frac{1}{12}\left[-A^{4}+33 A^{3}-94 A^{2}+74 A\right] \\
\Rightarrow & A^{-1}=\frac{1}{12}\left[-A^{3}+33 A^{2}-94 A+74 I\right]
\end{aligned}
$$

Now, $\mathrm{A}^{2}=\mathrm{A} \cdot \mathrm{A}$

$$
=\left(\begin{array}{cccc}
1 & 0 & 0 & 0 \\
1 & 1 & 1 & 1 \\
1 & 2 & 4 & 8 \\
1 & 3 & 9 & 27
\end{array}\right)\left(\begin{array}{cccc}
1 & 0 & 0 & 0 \\
1 & 1 & 1 & 1 \\
1 & 2 & 4 & 8 \\
1 & 3 & 9 & 27
\end{array}\right)
$$




$$
\begin{aligned}
\mathrm{A}^{3} & =\mathrm{A}^{2} . \mathrm{A} \\
& =\left(\begin{array}{cccc}
1 & 0 & 0 & 0 \\
4 & 6 & 14 & 36 \\
15 & 34 & 90 & 250 \\
40 & 102 & 282 & 804
\end{array}\right)\left(\begin{array}{cccc}
1 & 0 & 0 & 0 \\
1 & 1 & 1 & 1 \\
1 & 2 & 4 & 8 \\
1 & 3 & 9 & 27
\end{array}\right) \\
& =\left(\begin{array}{cccc}
1 & 0 & 0 & 0 \\
60 & 142 & 386 & 1090 \\
389 & 964 & 2644 & 7504 \\
1228 & 3078 & 8466 & 24066
\end{array}\right)
\end{aligned}
$$

$\therefore$ Equation (2.6.5) implies that

$$
\begin{aligned}
\mathrm{A}^{-1} & =\frac{1}{12}\left[-\mathrm{A}^{3}+33 \mathrm{~A}^{2}-94 \mathrm{~A}+74 \mathrm{I}\right] \\
& =\left(\begin{array}{cccc}
\frac{1}{-11} & 0 & 0 & 0 \\
6 & 3 & \frac{-3}{2} & \frac{1}{-3} \\
1 & \frac{-5}{2} & 2 & \frac{-1}{2} \\
\frac{-1}{6} & \frac{1}{2} & \frac{-1}{2} & \frac{1}{6}
\end{array}\right)
\end{aligned}
$$

Accordingly,

$$
X=A^{-1} B
$$

whence

$$
\begin{aligned}
\left(\begin{array}{l}
a_{0} \\
a_{1} \\
a_{2} \\
a_{3}
\end{array}\right) & =\left(\begin{array}{cccc}
1 & 0 & 0 & 0 \\
\frac{-11}{6} & 3 & \frac{-3}{2} & \frac{1}{3} \\
1 & \frac{-5}{2} & 2 & \frac{-1}{2} \\
\frac{-1}{6} & \frac{1}{2} & \frac{-1}{2} & \frac{1}{6}
\end{array}\right)\left(\begin{array}{c}
548159652 \\
683329097 \\
846302688 \\
1027015247
\end{array}\right) \\
& =\left(\begin{array}{c}
548159652 \\
117912312.66 \\
18934662 \\
-1677529.67
\end{array}\right)
\end{aligned}
$$

$\therefore a_{0}=548159652, a_{1}=117912312.66, a_{2}=18934662 \& a_{3}=-1677529.67$

$\therefore$ the Polynomial equations are 


$$
y=f(x)=548159652+117912312.66 x+18934662 x^{2}-1677529.67 x^{3}
$$

This polynomial yields,

$$
\begin{gathered}
f(0)=548159652, \\
f(1)=683329097, \\
f(2)=846302688 \\
\& f(3)=1027015247 .
\end{gathered}
$$

These are identical with the corresponding observed values.

\section{7. Method Based on Matrix Inversion by Elementary Transformation:}

Now solving these equations for the parameters, the polynomial can be obtained.

In order to solve the equations it is to be noted that the equations in (2.1) can be expressed as

$$
A C=B
$$

where

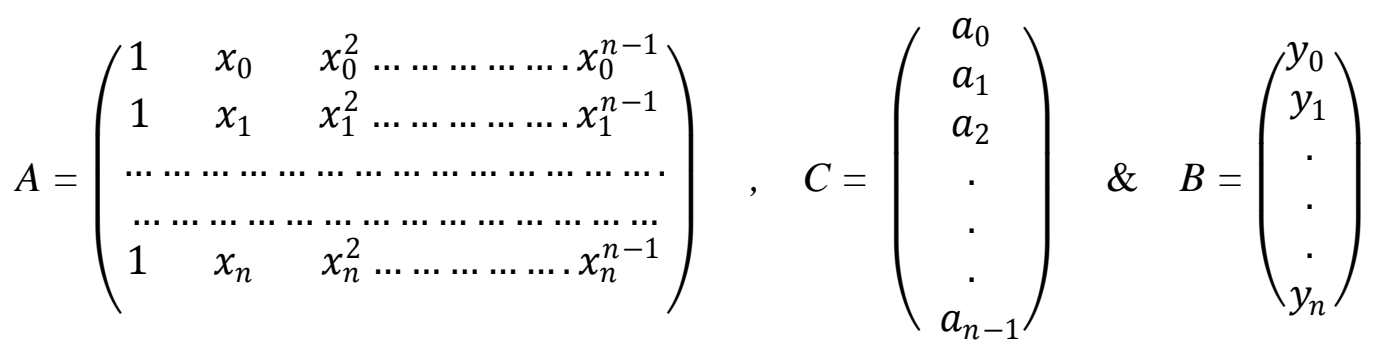

which gives

$$
C=A^{-1} B
$$

In order to find out $C$, it is required to find out $A^{-1}$.

To find out $A^{-1}$ one can apply Gauss Jordan method which is based on elementary row transformation of matrix [Cayley $(1858,1889$ et al) ; Hamilton (1889) et al].

Elementary Transformations (Operations) are the following operations on matrix:

(i) Interchange of two rows \& two columns

The interchange of $\mathrm{i}^{\text {th }} \& \mathrm{j}^{\text {th }}$ rows is denoted by $R_{i j}$

The interchange of $\mathrm{i}^{\text {th }} \& \mathrm{j}^{\text {th }}$ column is denoted by $C_{i j}$ 
(ii) Multiplication of (each element of) a row or column by a non zero number $\mathrm{k}$.

The multiplication of ith row by $\mathrm{k}$ is denoted by $\mathrm{k} R_{i}$

The multiplication of jth column by $\mathrm{k}$ is denoted by $\mathrm{k} C_{i}$

(iii) Addition of $\mathrm{k}$ times the elements of a row(or column) to the corresponding elements of another row (or column), $\mathrm{k} \neq 0$

The addition of $\mathrm{k}$ times the $\mathrm{j}^{\text {th }}$ row to the $\mathrm{i}^{\text {th }}$ row is denoted by $R_{i}+\mathrm{k} R_{j}$

The addition of $\mathrm{k}$ times the $\mathrm{j}^{\text {th }}$ row to the $\mathrm{i}^{\text {th }}$ row is denoted by $C_{i}+\mathrm{k} C_{j}$

If a matrix $\mathrm{B}$ is obtained from a matrix $\mathrm{A}$ by one or more $\mathrm{E}$ operation then $\mathrm{B}$ is said to be equivalent to $\mathrm{A}$ and is denoted by $\mathrm{A} \sim \mathrm{B}$.

The matrix obtained from the identity matrix I of order $n$ by applying it to any one of the Eoperations (elementary operations) is called an elementary matrix.

Gauss Jordan method of finding of $A^{-1}$ can be summarized as follows:

The elementary row (not column) operations which reduce a square matrix A to the unit matrix, give the inverse matrix $A^{-1}$

\section{Working rule:}

To find the inverse of A by E-row operations, we write A and I side by side and the same operations are performed on both. As soon as A is reduced to I, I will reduce to $A^{-1}$

\section{An Example of Application of the method:}

The following table shows the data on total population of India corresponding to the years:

\begin{tabular}{|c|c|c|c|c|}
\hline Year & 1971 & 1981 & 1991 & 2001 \\
\hline $\begin{array}{c}\text { Total } \\
\text { Population }\end{array}$ & 548159652 & 683329097 & 846302688 & 1027015247 \\
\hline
\end{tabular}

Taking 1971 as origin and changing scale by $1 / 10$, one can obtain the following table for independent variable $x$ (representing time) and $f(x)$ (representing total population of India):

\begin{tabular}{|c|c|c|c|c|}
\hline Year & 1971 & 1981 & 1991 & 2001 \\
\hline$x_{\mathrm{i}}$ & 0 & 1 & 2 & 3 \\
\hline$f\left(x_{i}\right)$ & 548159652 & 683329097 & 846302688 & 1027015247 \\
\hline
\end{tabular}


Here, $\quad x_{0}=0, x_{1}=1, x_{2}=2, x_{3}=3$,

$$
f\left(x_{0}\right)=548159652, f\left(x_{1}\right)=683329097, f\left(x_{2}\right)=846302688, f\left(x_{3}\right)=1027015247 \text {. }
$$

Thus, $\quad \mathrm{C}=\left(\begin{array}{l}a_{0} \\ a_{1} \\ a_{2} \\ a_{3}\end{array}\right), \mathrm{A}=\left(\begin{array}{cccc}1 & 0 & 0 & 0 \\ 1 & 1 & 1 & 1 \\ 1 & 2 & 4 & 8 \\ 1 & 3 & 9 & 27\end{array}\right), \quad \mathrm{B}=\left(\begin{array}{l}y_{0} \\ y_{1} \\ y_{2} \\ y_{3}\end{array}\right)$

Now,

$$
\mathrm{A}=\mathrm{IA}
$$

$$
\begin{aligned}
& \Rightarrow\left(\begin{array}{llll}
1 & 0 & 0 & 0 \\
1 & 1 & 1 & 1 \\
1 & 2 & 4 & 8 \\
1 & 3 & 9 & 27
\end{array}\right)=\left(\begin{array}{llll}
1 & 0 & 0 & 0 \\
0 & 1 & 0 & 0 \\
0 & 0 & 1 & 0 \\
0 & 0 & 0 & 1
\end{array}\right) \mathrm{A} \\
& \Rightarrow\left(\begin{array}{llll}
1 & 0 & 0 & 0 \\
0 & 1 & 1 & 1 \\
1 & 2 & 4 & 8 \\
1 & 3 & 9 & 27
\end{array}\right)=\left(\begin{array}{rrrr}
1 & 0 & 0 & 0 \\
-1 & 1 & 0 & 0 \\
0 & 0 & 1 & 0 \\
0 & 0 & 0 & 1
\end{array}\right) A, \quad \text { by } R_{2} \rightarrow R_{2}-R_{1} \\
& \Rightarrow\left(\begin{array}{llll}
1 & 0 & 0 & 0 \\
0 & 1 & 1 & 1 \\
0 & 2 & 4 & 8 \\
1 & 3 & 9 & 27
\end{array}\right)=\left(\begin{array}{rrrr}
1 & 0 & 0 & 0 \\
-1 & 1 & 0 & 0 \\
-1 & 0 & 1 & 0 \\
0 & 0 & 0 & 1
\end{array}\right) A, \quad \text { by } \quad R_{3} \rightarrow R_{3}-R_{1} \\
& \Rightarrow\left(\begin{array}{cccc}
1 & 0 & 0 & 0 \\
0 & 1 & 1 & 1 \\
0 & 2 & 4 & 8 \\
0 & 3 & 9 & 27
\end{array}\right)=\left(\begin{array}{cccc}
1 & 0 & 0 & 0 \\
-1 & 1 & 0 & 0 \\
-1 & 0 & 1 & 0 \\
-1 & 0 & 0 & 1
\end{array}\right) A, \quad \text { by } R_{4} \rightarrow R_{4}-R_{1} \\
& \Rightarrow\left(\begin{array}{llll}
1 & 0 & 0 & 0 \\
0 & 1 & 1 & 1 \\
0 & 1 & 2 & 4 \\
0 & 3 & 9 & 27
\end{array}\right)=\left(\begin{array}{cccc}
1 & 0 & 0 & 0 \\
-1 & 1 & 0 & 0 \\
-\frac{1}{2} & 0 & \frac{1}{2} & 0 \\
-1 & 0 & 0 & 1
\end{array}\right) A, \quad \text { by } \quad R_{3} \rightarrow \frac{1}{2} R_{3} \\
& \Rightarrow\left(\begin{array}{cccc}
1 & 0 & 0 & 0 \\
0 & 1 & 1 & 1 \\
0 & 0 & 1 & 3 \\
0 & 3 & 9 & 27
\end{array}\right)=\left(\begin{array}{cccc}
1 & 0 & 0 & 0 \\
-1 & 1 & 0 & 0 \\
\frac{1}{2} & -1 & \frac{1}{2} & 0 \\
-1 & 0 & 0 & 1
\end{array}\right) A, \quad \text { by } R_{3} \rightarrow R_{3}-R_{2} \\
& \Rightarrow\left(\begin{array}{llll}
1 & 0 & 0 & 0 \\
0 & 1 & 1 & 1 \\
0 & 0 & 1 & 3 \\
0 & 1 & 3 & 9
\end{array}\right)=\left(\begin{array}{cccc}
1 & 0 & 0 & 0 \\
-1 & 1 & 0 & 0 \\
\frac{1}{2} & -1 & \frac{1}{2} & 0 \\
-\frac{1}{3} & 0 & 0 & \frac{1}{3}
\end{array}\right) \text { A, by } \mathrm{R}_{4} \rightarrow \frac{1}{3} \mathrm{R}_{4}
\end{aligned}
$$




$$
\begin{aligned}
& \Rightarrow\left(\begin{array}{llll}
1 & 0 & 0 & 0 \\
0 & 1 & 1 & 1 \\
0 & 0 & 1 & 3 \\
0 & 0 & 2 & 8
\end{array}\right)=\left(\begin{array}{cccc}
1 & 0 & 0 & 0 \\
-1 & 1 & 0 & 0 \\
\frac{1}{2} & -1 & \frac{1}{2} & 0 \\
\frac{2}{3} & -1 & 0 & \frac{1}{3}
\end{array}\right) \text { A, by } \quad R_{4} \rightarrow R_{4}-R_{2} \\
& \Rightarrow\left(\begin{array}{cccc}
1 & 0 & 0 & 0 \\
0 & 1 & 1 & 1 \\
0 & 0 & 1 & 3 \\
0 & 0 & 1 & 4
\end{array}\right)=\left(\begin{array}{cccc}
1 & 0 & 0 & 0 \\
-1 & 1 & 0 & 0 \\
\frac{1}{2} & -1 & \frac{1}{2} & 0 \\
\frac{1}{3} & -\frac{1}{2} & 0 & \frac{1}{6}
\end{array}\right) A, \text { by } \quad \mathrm{R}_{4} \rightarrow \frac{1}{2} \mathrm{R}_{4} \\
& \Rightarrow\left(\begin{array}{llll}
1 & 0 & 0 & 0 \\
0 & 1 & 1 & 1 \\
0 & 0 & 1 & 3 \\
0 & 0 & 0 & 1
\end{array}\right)=\left(\begin{array}{rccc}
1 & 0 & 0 & 0 \\
-1 & 1 & 0 & 0 \\
\frac{1}{2} & -1 & \frac{1}{2} & 0 \\
-\frac{1}{6} & \frac{1}{2} & -\frac{1}{2} & \frac{1}{6}
\end{array}\right) \mathrm{A}, \quad \text { by } \quad \mathrm{R}_{4} \rightarrow \mathrm{R}_{4}-\mathrm{R}_{3} \\
& \Rightarrow\left(\begin{array}{cccc}
1 & 0 & 0 & 0 \\
0 & 1 & 1 & 1 \\
0 & 0 & \frac{1}{3} & 1 \\
0 & 0 & 0 & 1
\end{array}\right)=\left(\begin{array}{cccc}
1 & 0 & 0 & 0 \\
-1 & 1 & 0 & 0 \\
\frac{1}{6} & -\frac{1}{3} & \frac{1}{2} & 0 \\
-\frac{1}{6} & \frac{1}{2} & -\frac{1}{2} & \frac{1}{6}
\end{array}\right) \mathrm{A}, \quad \text { by } \quad \mathrm{R}_{3} \rightarrow \frac{1}{3} \mathrm{R}_{3} \\
& \Rightarrow\left(\begin{array}{cccc}
1 & 0 & 0 & 0 \\
0 & 1 & 1 & 1 \\
0 & 0 & -\frac{1}{3} & 0 \\
0 & 0 & 0 & 1
\end{array}\right)=\left(\begin{array}{rlll}
1 & 0 & 0 & 0 \\
-1 & 1 & 0 & 0 \\
-\frac{1}{3} & \frac{5}{6} & -\frac{2}{3} & \frac{1}{6} \\
-\frac{1}{6} & \frac{1}{2} & -\frac{1}{2} & \frac{1}{6}
\end{array}\right) A, \text { by } R_{3} \rightarrow R_{4}-R_{3} \\
& \Rightarrow\left(\begin{array}{cccc}
1 & 0 & 0 & 0 \\
0 & 1 & 1 & 0 \\
0 & 0 & -\frac{1}{3} & 0 \\
0 & 0 & 0 & 1
\end{array}\right)=\left(\begin{array}{cccc}
1 & 0 & 0 & 0 \\
-\frac{5}{6} & \frac{1}{2} & \frac{1}{2} & -\frac{1}{6} \\
-\frac{1}{3} & \frac{5}{6} & -\frac{2}{3} & \frac{1}{6} \\
-\frac{1}{6} & \frac{1}{2} & -\frac{1}{2} & \frac{1}{6}
\end{array}\right) A, \quad \text { by } R_{2} \rightarrow R_{2}-R_{4} \\
& \Rightarrow\left(\begin{array}{cccc}
1 & 0 & 0 & 0 \\
0 & 1 & 1 & 0 \\
0 & 0 & 1 & 0 \\
0 & 0 & 0 & 1
\end{array}\right)=\left(\begin{array}{cccc}
1 & 0 & 0 & 0 \\
-\frac{5}{6} & \frac{1}{2} & \frac{1}{2} & -\frac{1}{6} \\
-\frac{1}{3} & \frac{5}{6} & -\frac{2}{3} & \frac{1}{6} \\
-\frac{1}{6} & \frac{1}{2} & -\frac{1}{2} & \frac{1}{6}
\end{array}\right) A, \quad \mathrm{R}_{3} \rightarrow-3 \mathrm{R}_{3}
\end{aligned}
$$




$$
\begin{aligned}
\Rightarrow I & =\left(\begin{array}{cccc}
1 & 0 & 0 & 0 \\
-\frac{11}{6} & 3 & -\frac{3}{2} & \frac{1}{3} \\
1 & -\frac{5}{2} & 2 & -\frac{1}{2} \\
-\frac{1}{6} & \frac{1}{2} & -\frac{1}{2} & \frac{1}{6}
\end{array}\right) \text { A, by } R_{2} \rightarrow R_{2}-R_{3} \\
\Rightarrow A^{-1} & =\left(\begin{array}{cccc}
1 & 0 & 0 & 0 \\
-\frac{11}{6} & 3 & -\frac{3}{2} & \frac{1}{3} \\
1 & -\frac{5}{2} & 2 & -\frac{1}{2} \\
-\frac{1}{6} & \frac{1}{2} & -\frac{1}{2} & \frac{1}{6}
\end{array}\right)
\end{aligned}
$$

Therefore,

$$
\begin{aligned}
& X=A^{-1} B \\
&=\left(\begin{array}{cccc}
1 & 0 & 0 & 0 \\
\frac{-11}{6} & 3 & \frac{-3}{2} & \frac{1}{3} \\
1 & \frac{-5}{2} & 2 & \frac{-1}{2} \\
\frac{-1}{6} & \frac{1}{2} & \frac{-1}{2} & \frac{1}{6}
\end{array}\right)\left(\begin{array}{c}
548159652 \\
683329097 \\
846302688 \\
1027015247
\end{array}\right) \\
&=\left(\begin{array}{c}
548159652 \\
117912312.66 \\
18934662 \\
-1677529.67
\end{array}\right) \\
& \text { i.e. } \quad\left(\begin{array}{c}
a_{0} \\
a_{1} \\
a_{2} \\
a_{3}
\end{array}\right)=\left(\begin{array}{c}
548159652 \\
117912312.66 \\
18934662 \\
-1677529.67
\end{array}\right)
\end{aligned}
$$

Thus,

$$
a_{0}=548159652, a_{1}=117912312.66, a_{2}=18934662, a_{3}=-1677529.67
$$

Accordingly, the polynomial representing the given numerical data is

$$
y=548159652+117912312.66 x+18934662 x^{2}-1677529.67 x^{3}
$$

This polynomial yields the values of the function as follows:

$$
\begin{gathered}
f(0)=548159652, \\
f(1)=683329097,
\end{gathered}
$$




$$
\begin{aligned}
f(2) & =846302688 \\
\& f(3) & =1027015247 .
\end{aligned}
$$

These are identical with the corresponding observed values.

\subsection{Conclusion:}

The methods/formulae developed in the studies discussed here can be suitably used to represent a given set of numerical data on a pair of variables by a polynomial. The degree of the polynomial is one less than the number of pairs of observations.

The polynomial that represents the given set of numerical data can be used for interpolation at any position of the independent variable lying within its two extreme values.

The methods/formulae discussed here can be suitably applied in inverse interpolation also. These can be further applied in extrapolation with reasonable accuracy at a point with in a small interval outside of the range of the given values of the independent variable.

It has already been mentioned that in case of the interpolation by the existing formulae, if it is wanted to interpolate the values of the dependent variable corresponding to a number of values of the independent variable by a suitable existing interpolation formula then it is required to apply the formula for each value separately and thus the numerical computation of the value of the dependent variable based on the given data are to be performed in each of the cases. The methods/formulae developed in these studies have rescued from these repeated numerical computations from the given data. 


\section{References:}

Abramowitz M., "Handbook of Mathematical Functions with Formulas, Graphs, and Mathematical Tables", 9th printing, New York: Dover, pp. 880, 1972.

Bathe K. J. \& Wilson E. L., "Numerical Methods in Finite Element Analysis", Prentice-Hall, Englewood Cliffs, NJ, 1976.

Chakrabarty Dhritikesh, "Representation of Numerical Data on a Pair of Variables by a Polynomial Curve Expressed in the Simplest Form", International Journal of Electronics and Applied Research, Vol. 3, No. 1, pp. $26-39,2016 a$.

Chakrabarty Dhritikesh, "Interpolation: One Method of Representation of Numerical Data on a Pair of Variables by a Polynomial Curve Expressed In the Simplest Form", Journal of Environmental Science, Computer Science and Engineering \& Technology, Section C, Vol. 5, No. 3, pp. 405 - 418, 2016 b.

Chakrabarty Dhritikesh, "Difference and Ratio Operators: Representation of Numerical Data on a Pair of Variables by a Polynomial Curve", Journal of Environmental Science, Computer Science and Engineering \& Technology, Section C, Vol. 5, No. 4, pp. 549 -560, $2016 c$.

Chapra S. C. \& Canale R. P. , "Numerical Methods for Engineers", third ed., McGraw-Hill, NewYork, 2002.

Conte S. D. \& Boor Carl de, "Elementary Numerical Analysis", 3 Ed, McGraw-Hill, New York, USA, 1980.

Corliss J. J., "Note on an Extension of Lagrange's Formula", American Mathematical Monthly Jstor, Vol. 45, No. 2, pp. $106-107,1938$.

Cayley A., "A Memoir on the Theory of Matrices". Phil.Trans., Vol. 148, 1858.

Cayley A., "The Collected Mathematical Papers of Arthur Cayley (Classic Reprint)", Forgotten books, ASIN B008HUED9O, 1889.

Chwaiger J.,"On a Characterization of Polynomials by Divided Differences", Aequationes Math, Vol. 48, pp. $317-323,1994$.

Das Biswajit \& Chakrabarty Dhritikesh, "Lagranges Interpolation Formula: Representation of Numerical Data by a Polynomial Curve", International Journal of Mathematics Trend and Technology Vol. 34 (part1), No. 2, pp. $23-31,2016 a$.

Das Biswajit \& Chakrabarty Dhritikesh, "Newton's Divided Difference Interpolation Formula: Representation of Numerical Data by a Polynomial Curve", International Journal of Mathematics Trend and Technology, Vol. 35 (part-1), No. 3, pp. 26 - 32, $2016 b$.

Das Biswajit \& Chakrabarty Dhritikesh, "Newton's Forward Interpolation Formula: Representation of Numerical Data by a Polynomial Curve", International Journal of Applied Research, Vol. 1, No. 2, pp. 36 $-41,2016 c$. 
Das Biswajit \& Chakrabarty Dhritikesh, "Newton's Backward Interpolation Formula: Representation of Numerical Data by a Polynomial Curve", International Journal of Statistics and Applied Mathematics, Vol.2, No. 10, pp.513 - 517, 2016 d.

Das Biswajit \& Chakrabarty Dhritikesh, "Matrix Inversion: Representation of Numerical Data by a Polynomial curve", Aryabhatta Journal of Mathematics \& Informatics, Vol. 8, No. 2, pp. 267 - 276, $2016 e$.

Das Biswajit \& Chakrabarty Dhritikesh, "Inversion of Matrix by Elementary Transformation: Representation of Numerical Data by a Polynomial Curve", Journal of Mathematis and System Sciences, Vol. 12, No., pp. 27 - 32, $2016 f$.

De Boor C., "A divided difference expansion of a divided difference", J. Approx. Theory, Vol. 122, pp. $10-12,2003$.

Dokken T. \& Lyche T., "A divided difference formula for the error in Hermite interpolation”, BIT, Vol. 19, pp. $540-541,1979$.

Erdos P. \& Turan P., "On Interpolation II: On the Distribution of the Fundamental Points of Lagrange and Hermite Interpolation”, The Annals of Mathematics, 2nd Ser", Jstor, Vol. 39, No. 4, pp. 703 - 724, 1938.

Echols W. H., "On Some Forms of Lagrange's Interpolation Formula", "The Annals of Mathematics, Jstor, Vol. 8, No. 1, pp. 22 - 24, 1893.

Endre S. \& David M. , “An Introduction to Numerical Analysis”, Cambridge, UK, 2003.

Floater M. , "Error formulas for divided difference expansions and numerical differentiation", J. Approx. Theory, Vol. 122, pp. 1 - 9, 2003.

Fred T., "Recurrence Relations for Computing with Modified Divided Differences", Mathematics of Computation, Vol. 33, No. 148, pp. 1265 - 1271, 1979.

Gertrude B. , "On Modified Divided Differences", Mathematical Tables and Other Aids to Computation, Vol. 8, No. 45, pp. $1-11,1954$.

Gerald C. F. \& Wheatley P.O., "Applied Numerical Analysis”, fifth ed., Addison-Wesley Pub. Co., MA, 1994.

Herbert E. S. , "Multi-Point Generalization of Newton's Divided Difference Formula", Proceedings of the American Mathematical Society, Jstor, Vol. 13, No. 2, pp. 210 - 212, 1962.

Hummel P. M. , “A Note on Interpolation (in Mathematical Notes)", American Mathematical Monthly, Jstor, Vol. 54, No. 4, pp. 218 - 219, 1947.

Hamilton W. R. , “Lectures on Quaternions”, Dublin , 1853.

Hamilton W. R. , "On the Existence of a Symbolic and Biquadratic Equation which is satisfied by the Symbol of Linear or Distributive Operation on a Quaternion", The London, Edinburgh and Dublin Philosophical Magazine and Journal of Science, Series IV, Taylor \& Francis 24, pp. 127 - 128, 1862. 
Hamilton W. R. , "On a New and General Method of Inverting a Linear and Quaternion Function of a Quaternion", Proceedings of the Royal Irish Academy, Royal Irish Academy, Vol. VIII, pp. 182 - 183, $1864 a$.

Hamilton W. R. , "On the Existence of a Symbolic and Biquadratic Equation, which is satisfied by the Symbol of Linear Operation in Quaternions", Proceedings of the Royal Irish Academy, Royal Irish Academy, Vol. VIII, pp. 190-101, 1864 b.

Jordan C. , "Calculus of Finite Differences", 3rd ed, New York, Chelsea, 1965.

Jeffreys H. \& Jeffreys B. S. , "Divided Differences”, Methods of Mathematical Physics, $3^{\text {rd }}$ ed, pp. 260 264, 1988.

Jan K. W. , "Note on Interpolation (in Notes)", Journal of the American Statistical Association, Jstor, Vol. 25, 170, pp. $203-205,1930$.

John H. M. \& Kurtis D.F. , "Numerical methods using MATLAB”, 4ed, Pearson Education, USA, 2004.

Kincard David R. \& Ward Chaney E., "Numerical analysis”, Brooks /Cole, Pacific Grove, CA, 1991.

Kendall E. Atkinson, “An Introduction to Numerical Analysis”, $2^{\text {nd }}$ Ed., New York, 1989.

Lee E.T.Y. , “A Remark on Divided Differences”, American Mathematical Monthly, Vol. 96, No 7, pp. $618-622,1989$.

Mills T. M., "An introduction to analysis by Lagrange interpolation", Austral. Math. Soc. Gaz., MathSciNet, Vol. 4, No. 1, pp. 10 - 18, 1977.

Nasrin Akter Ripa, "Analysis of Newton's Forward Interpolation Formula", International Journal of Computer Science \& Emerging Technologies, Vol. 1, No. 4, 2010.

Neale E. P. \& Sommerville D. M. Y. , “A Shortened Interpolation Formula for Certain Types of Data”, Journal of the American Statistical Association, Jstor, Vol. 19, No. 148, pp. 515 - 517, 1924.

Quadling D. A. , "Lagrange's Interpolation Formula", The Mathematical Gazette, Vol. L, No. 374, pp. $372-375,1966$.

Robert J. Schilling \& Sandra L. Harries, “Applied Numerical Methods for Engineers”, Brooks /Cole, Pacific Grove, CA, 2000.

Revers \& Michael Bull, "On Lagrange interpolation with equally spaced nodes", Austral. Math. Soc, MathSciNet., Viol. 62, No. 3, pp. 357 - 368, 2000.

Scarborough James B. , "Numerical Mathematical Analysis", 6 Ed, The John Hopkins Press, USA, 1996.

Traub J. F., "On Lagrange-Hermite Interpolation", Journal of the Society for Industrial and Applied Mathematics, Jstor, Vol. 12, No. 4, pp. 886-891, 1964.

Vertesi P., "SIAM Journal on Numerical Analysis", Jstor, Vol. 27, No. 5, pp. 1322-1331, 1990. 
Whittaker E. T. and Robinson G., "The Gregory-Newton Formula of Interpolation" and "An Alternative Form of the Gregory-Newton Formula", "The Calculus of Observations: A Treatise on Numerical Mathematics", 4th ed, pp. $10-15,1967 a$.

Whittaker E. T. \& Robinson G., "Divided Differences \& Theorems on Divided Differences", "The Calculus of Observations: A Treatise on Numerical Mathematics", $4^{\text {th }}$ ed., New York, pp. $20-24,1967 b$. 